\title{
A New Approach to the Solution of Robot Kinematics Based on Relative Transformation Matrices
}

\author{
Mohammad Reza Elhami, Iman Dashti \\ Departement of Mechanical Engineering, Imam Hosein University, Iran
}

\begin{tabular}{l} 
Article Info \\
\hline Article history: \\
Received Jun 2, 2016 \\
Revised Aug 6, 2016 \\
Accepted Aug 20, 2016 \\
\hline Keyword: \\
Convenient approach \\
Denavit hartenberg \\
Relative transformation \\
Principle \\
Robot kinematics
\end{tabular}

\begin{abstract}
In analyzing robot manipulator kinematics, we need to describe relative movement of adjacent linkages or joints in order to obtain the pose of end effector (both position and orientation) in reference coordinate frame. Denavit-Hartenberg established a method based on a $4 \times 4$ homogenous matrix so called "A" matrix. This method used by most of the authors for kinematics and dynamic analysis of the robot manipulators. Although it has many advantages, however, finding the elements of this matrix and link/joint's parameters is sometimes complicated and confusing. By considering these difficulties, the authors proposed a new approach called 'convenient approach' that is developed based on "Relative Transformations Principle". It provides a very simple and convenient way for the solution of robot kinematics compared to the conventional D-H representation. In order to clarify this point, the kinematics of the well-known Stanford manipulator has been solved through D-H representation as well as convenient approach and the results are compared.
\end{abstract}

Copyright $\odot 2016$ Institute of Advanced Engineering and Science. All rights reserved.

\section{Corresponding Author:}

Mohammad Reza Elhami,

Departement of Mechanical Engineering,

Imam Hosein University,

Babaei Highway, Tehran, Iran,

Email: melhami@ihu.ac.ir

\section{INTRODUCTION}

Kinematic analysis is a fundamental part of mechanism design and analysis that has a long history of establishment. Reuleaux [1] developed a symbolic method of describing the kinematic properties of a mechanism. Denavit and Hartenberg [2] subsequently obtained a new form of kinematic notation representing all possible forms of lower-pair mechanism. This new representation expressed in terms of a $4 \times 4$ homogeneous transformation matrix and the effect of a number of coupled links was determined from the product of a number of matrices. Paul and Shimano [3] recognized that the effect of a single joint and its associated link on a robot could be described by four parameters and obtained a single homogenous matrix (the A matrix) representing these parameters. Forward and inverse kinematic solutions derived for the Stanford Arm. Subsequently Paul, Shimano and Mayor [4] illustrated the method by deriving solutions for Puma manipulator. Lee [5] and Fu, Gonzalez and Lee [7] used the similar method to determine coordinate transformations for a Puma robot to perform kinematics and dynamics analysis. This transformation method then established as a standard tool for all authors in robot kinematics. This standard homogenous transformation approach has established four parameters describing each joint and the associated link I as follows: the joint angle $\theta_{i}$, the link length $a_{i}$, the link offset $d_{i}$, the joint twist $\alpha_{i}$

Determination of these parameters could be difficult, particularly for ones who unfamiliar with robotics, this problem becomes worse in determining the joint twist while one or more link lengths are zero. This paper is intended to suggest a simplified method to obtain the A matrices based on "Relative Transformations Principle". The application of this convenient approach is illustrated by performing the forward kinematic analysis on Stanford manipulators to obtain the end effector position and orientation vectors. 


\section{DENAVIT-HARTENBERG METHOD}

Explaining research chronological, including research design, research procedure (in the form of algorithms, Pseudocode or other), how to test and data acquisition [1]-[3]. The description of the course of research should be supported references, so the explanation can be accepted scientifically [2], [4].

Denavit-Hartenberg method represents each transformation by a specific convention established by a series of definitions. Consider a robot manipulator with $\mathrm{n}$ kinematic pairs (rotated and prismatic). Let Li the $\mathrm{i}$-th link and ji the $\mathrm{i}$-th kinematic pair between $\mathrm{L}_{\mathrm{i}-1}$ and $\mathrm{L}_{\mathrm{i}}, \mathrm{i}=1,2, \ldots, \mathrm{n}$. $\mathrm{L} 0$ is the link between base and the first kinematic pair. After defining a reference coordinate system, one coordinate system assigned for each joint of robot manipulator, also in the end-effector, in order to establish the coordinate transformation between links, and solve the robotic kinematics. In summary, Denavit-Hartenberg method defines a frame $\mathrm{F}_{\mathrm{i}}$, $\mathrm{i}=0, \ldots, \mathrm{n}$, by:

- $\quad \mathrm{z}_{\mathrm{i}}$-axis: axis of the $\mathrm{i}+1$ joint;

- $\mathrm{x}_{\mathrm{i}}$-axis: is parallel to the common normal: $x_{i}=z_{i-1} \times z_{i}$;

- $\mathrm{y}_{\mathrm{i}}$-axis: follows from right-hand rule;

- $\quad O i$ : intersection between $\mathrm{z}_{\mathrm{i}}$ axis and common normal;

- $O_{i}^{\prime}$ : intersection between $\mathrm{z}_{\mathrm{i}-1}$ axis and common normal;

Then, a transformation from frame $\mathrm{F}_{\mathrm{i}}$ to frame $\mathrm{F}_{\mathrm{i}-1}$ defined by $\mathrm{DH}$ parameters:

- $\quad \mathrm{a}_{\mathrm{i}}$ : distance from $O i$ and $O_{i}^{\prime}$ measured along common normal;

- $\quad \mathrm{d}_{\mathrm{i}}$ : distance from $O_{i-1}$ and $O_{i}^{\prime}$, measured along $\mathrm{z}_{\mathrm{i}}$;

- $\quad \alpha_{i}$ : angle between axes $\mathrm{z}_{\mathrm{i}-1}$ and $\mathrm{z}_{\mathrm{i}}$ about axis $\mathrm{x}_{\mathrm{i}}$ to be taken positive when rotation is made counter-clockwise;

- $\quad \theta_{i}$ :angle between axes $\mathrm{x}_{\mathrm{i}-1}$ and $\mathrm{xi}$ about axis $\mathrm{z}_{\mathrm{i}-1}$ to be taken positive when rotation is made counter-clockwise;

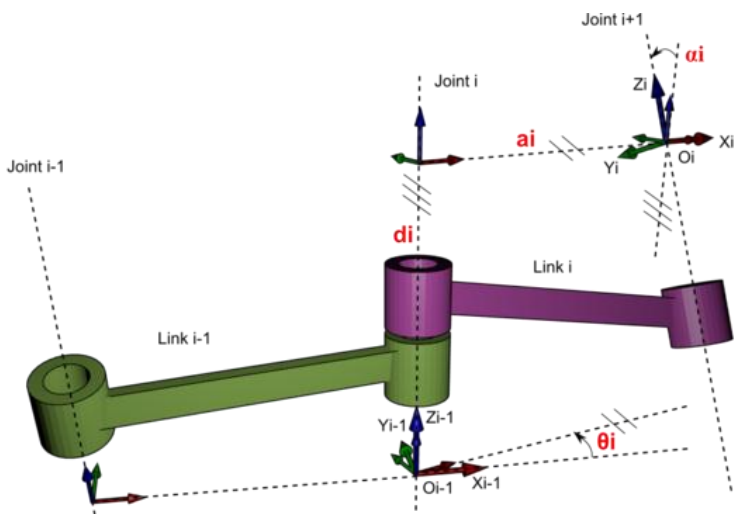

Figure 1. D-H parameters definition

More about Denavit Hartenberg convention can be found in [6,12,14].

In general, D-H parameters can be tabulated, leaving the transformations as functions of the variable $\theta$, in the revolute case, or $\mathrm{d}$, in the prismatic case. Homogeneous coordinates allow establishing the relation between two adjacent links, connected by a kinematic pair, following four steps:

$$
\text { Rotate } \alpha \text { in } O_{x} \rightarrow \text { Translate a by } O_{x} \rightarrow \text { Rotate } \theta \text { in } O_{z} \rightarrow \text { Translate din } O_{z}
$$

The steps above express the product of four homogeneous transformation matrix from frame $F_{i}$ to frame $F_{i-1}$, $i=1, \ldots, n$, that is 


$$
\begin{aligned}
& A_{i}^{i-1}=T_{z}\left(d_{i}\right) R_{z}\left(\theta_{i}\right) T_{x}\left(a_{i}\right) R_{x}\left(\alpha_{i}\right) \\
& =\left[\begin{array}{cccc}
\cos \theta_{i} & -\cos \alpha_{i} \sin \theta_{i} & \sin \alpha_{i} \sin \theta_{i} & a_{i} \cos \theta_{i} \\
\sin \theta_{i} & \cos \alpha_{i} \cos \theta_{i} & -\sin \alpha_{i} \cos \theta_{i} & a_{i} \sin \theta_{i} \\
0 & \sin \alpha_{i} & \cos \alpha_{i} & d_{i} \\
0 & 0 & 0 & 1
\end{array}\right]
\end{aligned}
$$

\section{THE CONVENIENT APPROACH}

\subsection{Coordinate Frames}

A homogenous transformation matrix can be used to describe (oxyz) frame with respect to a reference coordinate frame (oxyz). The first three rows of this $4 * 4$ matrix are composed of a $3 * 1 \mathrm{O}$ vector (denoting the position of $(o x y z)^{\prime}$ origin in (oxyz) frame) and a $3 * 3$ rotation $\mathrm{R}$ matrix (denoting the orientation of $(o x y z)^{\prime}$ axes in (oxyz) frame).

$$
T_{O x y z}^{O x y z}=\left[\begin{array}{cccc}
{ }^{O x y z} & & { }^{O x y z} \\
{ }^{O x y z} & { }^{O x y z} O_{3 * 1} \\
0 & 0 & 0 & 1
\end{array}\right]
$$

This is illustrated through an example. Consider a coordinate frame, i.e. $(o x y z)^{\prime}$, specified by:

$$
T_{\text {Oxyz }}^{\text {Oxyz }}=\left[\begin{array}{cccc}
0 & 0 & -1 & -1 \\
1 & 0 & 0 & 3 \\
0 & -1 & 0 & 2 \\
0 & 0 & 0 & 1
\end{array}\right]
$$

The rotation and position matrices are as follows:

$$
\begin{gathered}
\underset{O^{\prime} y z}{ } R=\left[\begin{array}{ccc}
0 & 0 & -1 \\
1 & 0 & 0 \\
0 & -1 & 0
\end{array}\right] \\
\underset{O_{\text {Oxyz }} O}{O}=\left[\begin{array}{c}
-1 \\
3 \\
2
\end{array}\right]
\end{gathered}
$$

Thus, the position and direction of $(o x y z)^{\prime}$ frame with respect to (oxyz) frame are expressed as:

$$
\begin{aligned}
& \vec{i}^{\prime}=\left[\begin{array}{llll}
0 & 1 & 0 & 0
\end{array}\right]^{T} \\
& \vec{j}^{\prime}=\left[\begin{array}{llll}
0 & 0 & -1 & 0
\end{array}\right]^{T} \\
& \vec{k}^{\prime}=\left[\begin{array}{llll}
-1 & 0 & 0 & 0
\end{array}\right]^{T} \\
& \vec{o}^{\prime}=\left[\begin{array}{llll}
-1 & 3 & 2 & 1
\end{array}\right]^{T}
\end{aligned}
$$

And therefore, the origin of $(O X Y Z)^{\prime}$ is located on the point of $(-1,3,2)$ and direction of $X^{\prime}, Y^{\prime}, Z^{\prime}$ axes are along $Y,-Z,-X$ direction of original frame, respectively. Consequently, $(O X Y Z)^{\prime}$ frame could be outlined in a very simple manner. 


\subsection{Geometric Interpretation of homogenous Transformation Matrix (HTM), T:}

Describing geometric interpretation of Homogenous transformation matrix, T, let's have a look at preceding example from another angle. Consider the ways which $(O X Y Z)^{\prime}$ frame is reached to its current state from its initial state with respect to the base frame $(O X Y Z)$. There are two ways for the solution of this problem: we can either first translate the origin of $(O X Y Z)^{\prime}$ frame to the desired position i.e. $\left[\begin{array}{llll}-1 & 3 & 2 & 1\end{array}\right]^{T}$, then rotate it about $Y$ and $Z$ axes of current frame respectively; Or first rotate it about $\mathrm{Y}$ and $\mathrm{X}$ axes of base frame respectively, then translate it to the desired position, i.e. $\left[\begin{array}{cccc}-1 & 3 & 2 & 1\end{array}\right]^{T}$.

Since these two ways lead to different order of matrix multiplication and matrix multiplication is non-commutative, there comes a main problem that in what order these matrices are multiplied in order to get the same result.

\subsection{Relative Transformations Principle (RTP)}

There is a fundamental rule behind the order of multiplication, which is called "Relative Transformation Principle" [8]. This principle states where we can multiply the matrices in the order of transformation operation or do it in the reverse direction.

Multiplication of the matrices in the order of transformation operation so called Post-multiplication, means that transformation refers to the Current Coordinate Frame; and multiplication in the reverse order of operations is called Pre-multiplication and means that transformation referred w.r.t. Base or Reference Coordinate Frame. Therefore, there is a "Pre-Base; Post-Current" resolution for this principle. To clarify this point, let's have a look at the previous example again. There are two approaches for frame (XYZ) or BaseFrame to reach frame $(X Y Z)^{\prime}$ or Current Frame as shown in figure 2.

\section{- Post-Current}

$$
\begin{gathered}
\operatorname{Trans}(-1,3,2) \operatorname{Rot}\left(Y,-90^{\circ}\right) \operatorname{Rot}\left(Z^{\prime}, 90^{\circ}\right)= \\
{\left[\begin{array}{cccc}
1 & 0 & 0 & -1 \\
0 & 1 & 0 & 3 \\
0 & 0 & 1 & 2 \\
0 & 0 & 0 & 1
\end{array}\right]\left[\begin{array}{cccc}
0 & 0 & -1 & 0 \\
0 & 1 & 0 & 0 \\
1 & 0 & 0 & 0 \\
0 & 0 & 0 & 1
\end{array}\right]\left[\begin{array}{cccc}
0 & -1 & 0 & 0 \\
1 & 0 & 0 & 0 \\
0 & 0 & 1 & 0 \\
0 & 0 & 0 & 1
\end{array}\right]} \\
=\left[\begin{array}{cccc}
0 & 0 & -1 & -1 \\
1 & 0 & 0 & 3 \\
0 & -1 & 0 & 2 \\
0 & 0 & 0 & 1
\end{array}\right]
\end{gathered}
$$

- Pre-Base

$$
\begin{aligned}
& \operatorname{Trans}(-1,3,2) \operatorname{Rot}\left(X,-90^{\circ}\right) \operatorname{Rot}\left(Y,-90^{\circ}\right)= \\
& {\left[\begin{array}{cccc}
1 & 0 & 0 & -1 \\
0 & 1 & 0 & 3 \\
0 & 0 & 1 & 2 \\
0 & 0 & 0 & 1
\end{array}\right]\left[\begin{array}{cccc}
1 & 0 & 0 & 0 \\
0 & 0 & 1 & 0 \\
0 & -1 & 0 & 0 \\
0 & 0 & 0 & 1
\end{array}\right]\left[\begin{array}{cccc}
0 & 0 & -1 & 0 \\
0 & 1 & 0 & 0 \\
1 & 0 & 0 & 0 \\
0 & 0 & 0 & 1
\end{array}\right]} \\
& =\left[\begin{array}{cccc}
0 & 0 & -1 & -1 \\
1 & 0 & 0 & 3 \\
0 & -1 & 0 & 2 \\
0 & 0 & 0 & 1
\end{array}\right]
\end{aligned}
$$

It is shown that although there are two different approaches and eventually two different matrix equations, however, the results are the same. (See Figure 2). 


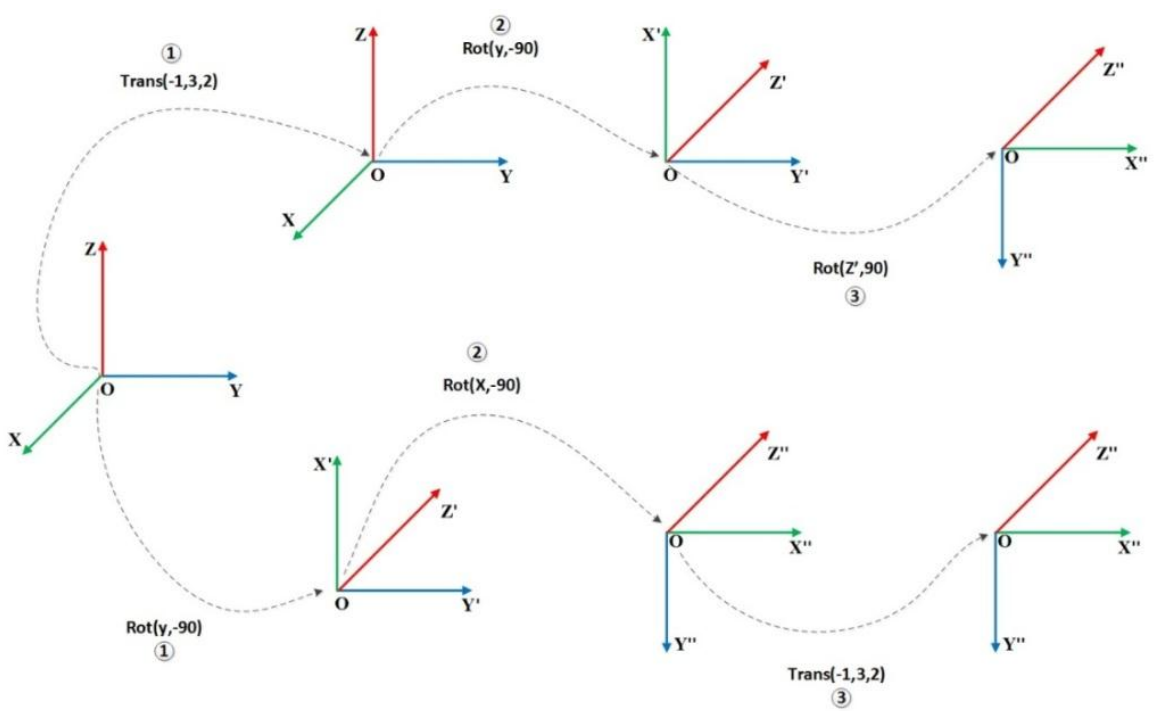

Figure 2. Relative Transformation Principle

\subsection{The Convenient Approach}

In analysis of robot kinematics, every link/joint pair attributed with a ${ }^{i-1} A_{i}$ matrix, which describes its pose (both position and direction) relative to the previous one. In the previous section, the "Pre-Base PostCurrent" resolution introduced. To derive a ${ }^{i-1} A_{i}$ matrix there are only two rules to be applied:

1) The $Z_{i}$ axis lies along the axis of joint i's movement, $X_{i}$ and $Y_{i}$ axes directions are optional, just satisfy the right-hand rules

2) By using relative transformations principle, transformation matrix of link/joint $i$ with respect to joint $i-1$, which is composed of fixed and variable kinematic parameters, could be obtained directly and very straightforward.

In fact, every A matrix is produced by multiplication of two matrices. One is a fixed HTM matrix and the other is a variable HTM matrix. The fixed HTM matrix describes the original geometric structure of the link and is derived by substituting the relative direction of $\mathrm{X}, \mathrm{Y}$ and $\mathrm{Z}$ axes and relative position of joint $\mathrm{i}$ origin frame's (P vector) into i-1 coordinate frame. Just like equations (3)-(6) in the previous section example, we can derive them instantly and put them in the same order of columns very simple.

Another one, the variable HTM matrix is a function of joint variable parameter and characterizes the relative movement allowed at each joint and is only a rotation or translation about/along $\mathrm{Z}$ axis that covers $\theta_{i}$ and $d_{i}$ parameters in revolute and prismatic joints, respectively. The transformation matrix for each joint is then obtained by multiplication of these two matrices, which in post-current approach is as:

$$
A=F^{*} V
$$

and in pre-base approach is:

$$
A=V^{*} F
$$

Considering F and V as fixed and variable HTM of each joint respectively. It is worth to notice that for convenience usually "Post-current" part of the resolution is used to derive the A matrix for entire kinematic equations from base frame to end effector to describe the forward kinematic of robot arms.

\section{COMPARISON OF THE TWO METHODS: D-H NOTATION AND CONVENIENT APPROACH}

D-H notation has established a very efficient and extensive method to describe the HTM of every link in any manipulator. It is also a concise model of every kind of link/joint pair in any mechanism. Although it has some advantages so that has become the most popular robot kinematics analysis method in literature, there are many difficulties and complications in the process of parameters determination. 
The main difficulties arises in recognition of the difference between $d_{i}$ and $a_{i}$ or $\theta_{i}$ and $\alpha_{i}$ as well as ambiguity in the definition of the sign of $\alpha_{i}$, especially when robot has different configurations which makes the substitution of parameters in A matrix a time-consuming calculation. These disadvantages cause major problems for development of robot dynamic control system in industry and popularity of robotics in education system, mainly for the students who are in the first stages of learning robotics.

The Convenient Approach, which is proposed in this paper has considered these problems and offered a simplified method to obtain the A matrix to determine kinematic configuration of each link/joint pair of robot arms. This method is straightforward, has more clarity in concept and less complexity in computation; therefore it has easy implementation in kinematic control and easy understanding for education of robotics.

To get familiar with this method, it is only required to understand the core concept of Relative Transformations Principle (RTP) and its Pre-Current Post-Base resolution to derive A matrix very easily and directly. There is no more anxious about identification and determination of those four link/joint parameters. For computation matter there is merely the need to know the rotation/translation matrices about Z-axis and matrix multiplication rules.

The main privilege of this method can be shown in most robots, especially industrial robots for convenience in analysis, especially dynamic analysis, and eventually simplicity in manufacturing, they are designed with $\alpha_{i}=0$ or $\pm \pi$ in robot arms. Therefore, derivation of fixed HTM of A matrix becomes very handy and straightforward. However, for $\alpha_{i}$ in any condition, thanks to the concept clearness and algorithm simplicity, this approach is still applicable compared to D-H notation.

\section{THE ILLUSTRATION METHOD}

To show that the convenient approach is applicable to the real and standard industrial robots, the implementation of this method is illustrated by analyzing the forward kinematic to obtain the end effector pose vector for Stanford manipulator. This manipulator is an example of a spherical (RRP) manipulator with a spherical wrist. It has an offset in the shoulder joint that slightly complicates both the forward and inverse kinematics.

\subsection{D-H Representation}

We first establish the joint coordinate frames using the D-H conventions as shown in Figure 3. The ${ }^{i} A_{i-1}$ matrix for each joints in D-H method is as follows:

$$
A_{i-1}^{i}=\left[\begin{array}{cccc}
C_{\theta_{i}} & -C_{\alpha_{i}} C_{\theta_{i}} & S_{\alpha_{i}} S_{\theta_{i}} & a_{i} C_{\theta_{i}} \\
S_{\theta_{i}} & C_{\alpha_{i}} C_{\theta_{i}} & -S_{\alpha_{i}} C_{\theta_{i}} & a_{i} S_{\theta_{i}} \\
0 & S_{\alpha_{i}} & C_{\alpha_{i}} & d_{i} \\
0 & 0 & 0 & 1
\end{array}\right]
$$

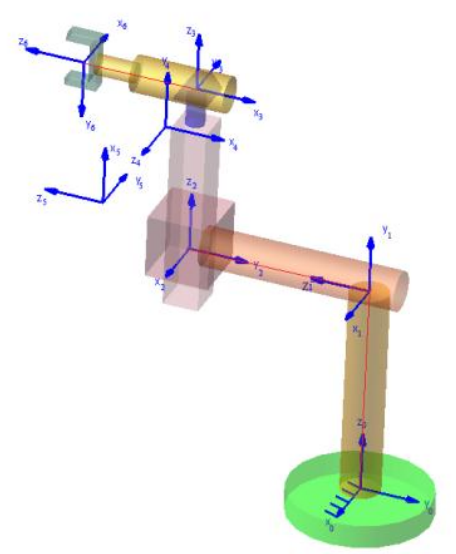

Figure 3. Stanford Robot and D-H Representation for its Link Coordinate System

D-H parameters for Stanford link coordinate system shown in Table1. 
Table 1. Establishing link coordinate system for Stanford Manipulator[6]

\begin{tabular}{ccccc}
\hline Joint $\boldsymbol{i}$ & $\theta_{i}$ & $\alpha_{i}$ & $a_{i}$ & $d_{i}$ \\
\hline 1 & $\theta_{1}$ & $90^{\circ}$ & 0 & $h_{1}$ \\
2 & $\theta_{2}$ & $-90^{\circ}$ & 0 & $h_{2}$ \\
3 & 90 & 0 & 0 & $d_{3}$ \\
4 & $\theta_{4}$ & $90^{\circ}$ & 0 & 0 \\
5 & $\theta_{5}+90$ & $-90^{\circ}$ & 0 & 0 \\
6 & $\theta_{6}+90$ & 0 & 0 & $h_{6}$
\end{tabular}

Transformation matrices from the base to the end effector i.e. ${ }^{i-1} A_{i}$ of link/joint i relative to joint i- 1 could be obtained as (12):

$$
\begin{gathered}
A_{1}^{0}=\left[\begin{array}{cccc}
C_{1} & 0 & S_{1} & 0 \\
S_{1} & 0 & -C_{1} & 0 \\
0 & 1 & 0 & h_{1} \\
0 & 0 & 0 & 1
\end{array}\right], A_{2}^{1}=\left[\begin{array}{cccc}
C_{2} & 0 & -S_{2} & 0 \\
S_{2} & 0 & C_{2} & 0 \\
0 & -1 & 0 & h_{2} \\
0 & 0 & 0 & 1
\end{array}\right], A_{3}^{2}=\left[\begin{array}{cccc}
0 & -1 & 0 & 0 \\
1 & 0 & 0 & 0 \\
0 & 0 & 1 & d_{3} \\
0 & 0 & 0 & 1
\end{array}\right] \\
A_{4}^{3}=\left[\begin{array}{cccc}
C_{4} & 0 & S_{4} & 0 \\
S_{4} & 0 & -C_{4} & 0 \\
0 & 1 & 0 & 0 \\
0 & 0 & 0 & 1
\end{array}\right], A_{5}^{4}=\left[\begin{array}{cccc}
-S_{5} & 0 & -C_{5} & 0 \\
C_{5} & 0 & -S_{5} & 0 \\
0 & -1 & 0 & 0 \\
0 & 0 & 0 & 1
\end{array}\right], A_{6}^{5}=\left[\begin{array}{cccc}
-S_{6} & -C_{6} & 0 & 0 \\
C_{6} & -S_{6} & 0 & 0 \\
0 & 0 & 1 & h_{6} \\
0 & 0 & 0 & 1
\end{array}\right]
\end{gathered}
$$

Hence, the HTM of end effector ${ }^{0} T_{6}$ is:

$$
T_{6}^{0}=A_{1}^{0} A_{2}^{1} A_{3}^{2} A_{4}^{3} A_{5}^{4} A_{6}^{5}=\left[\begin{array}{cccc}
n_{x} & s_{x} & a_{x} & p_{x} \\
n_{y} & s_{y} & a_{y} & p_{y} \\
n_{z} & s_{z} & a_{z} & p_{z} \\
0 & 0 & 0 & 1
\end{array}\right]
$$

Where

$$
\begin{gathered}
n_{x}=C_{6}\left[S_{4} S_{1}-C_{4} C_{2} C_{1}\right]-S_{6}\left[S_{5} C_{4} S_{1}+C_{1} C_{2} S_{4}\right]-C_{1} C_{5} S_{\partial 2}, \\
n_{y}=S_{6}\left[S_{5} C_{1} C_{4}-S_{1} C_{2} S_{4}+C_{5} S_{1} S_{2}\right]-C_{6}\left[S_{4} C_{1}-C_{2} S_{1} C_{4}\right], \\
n_{z}=-S_{6}\left[C_{2} C_{5}+S_{2} S_{4} S_{5}\right]-S_{2} C_{4} C_{6} \\
s_{x}=-C_{6}\left[S_{5}\left(C_{4} S_{1}+S_{4} C_{1} C_{2}\right)-C_{1} C_{5} S_{2}\right]-S_{6}\left[S_{4} S_{1}-C_{1} C_{2} C_{4}\right], \\
s_{y}=C_{6}\left[S_{5}\left(C_{4} C_{1}-S_{4} C_{2} S_{1}\right)+S_{2} C_{5} S_{1}\right]+S_{6}\left[S_{4} C_{1}+C_{4} C_{2} S_{1}\right], \\
s_{z}=-C_{6}\left[C_{2} C_{5}+S_{2} S_{5} S_{4}\right]+S_{2} C_{4} S_{6} \\
a_{x}=C_{5}\left(S_{1} C_{4}+C_{1} S_{4} C_{2}\right)+C_{1} S_{2} S_{5} \\
a_{y}=S_{1} S_{2} S_{5}-C_{5}\left(C_{1} C_{4}-C_{2} S_{1} S_{4}\right) \\
a_{z}=S_{2} S_{4} C_{5}-C_{2} S_{5} \\
p_{x}=h_{2} S_{1}+h_{6}\left[C_{5}\left(C_{4} S_{1}+C_{1} C_{2} S_{4}\right)+C_{1} S_{2} S_{5}\right]-d_{3} C_{1} S_{2} \\
p_{y}=-h_{2} C_{1}-h_{6}\left[C_{5}\left(C_{1} C_{4}-C_{2} S_{1} S_{4}\right)-S_{1} S_{2} S_{5}\right]-d_{3} S_{1} S_{2} \\
p_{z}=h_{1}-h_{6}\left(C_{2} S_{5}-C_{5} S_{2} S_{4}\right)+d_{3} C_{2}
\end{gathered}
$$




\subsection{The Convenient Approach}

The coordinate frame system used for Post-current resolution of convenient method shown in Figure 4. Notice that we have a different frame at each joint, as zi axis must lie along the joint $\theta_{i}$ or di for revolute and prismatic joint, respectively.

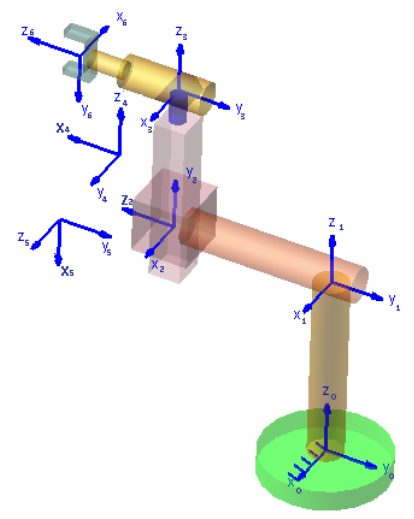

Figure 4. Stanford Link Coordinate System in Post-current Approach

Transformation matrices obtained as follows:

$$
\begin{aligned}
& \underline{A_{1}^{0}}=\left[\begin{array}{cccc}
1 & 0 & 0 & 0 \\
0 & 1 & 0 & 0 \\
0 & 0 & 1 & h_{1} \\
0 & 0 & 0 & 1
\end{array}\right]\left[\begin{array}{cccc}
C_{1} & -S_{1} & 0 & 0 \\
S_{1} & C_{1} & 0 & 0 \\
0 & 0 & 1 & 0 \\
0 & 0 & 0 & 1
\end{array}\right]=\left[\begin{array}{cccc}
C_{1} & S_{1} & 0 & 0 \\
S_{1} & C_{1} & 0 & 0 \\
0 & 0 & 1 & h_{1} \\
0 & 0 & 0 & 1
\end{array}\right] \\
& \underline{A_{2}^{1}}=\left[\begin{array}{cccc}
1 & 0 & 0 & 0 \\
0 & 0 & -1 & -h_{2} \\
0 & 1 & 0 & 0 \\
0 & 0 & 0 & 1
\end{array}\right]\left[\begin{array}{cccc}
C_{2} & -S_{2} & 0 & 0 \\
S_{2} & C_{2} & 0 & 0 \\
0 & 0 & 1 & 0 \\
0 & 0 & 0 & 1
\end{array}\right]=\left[\begin{array}{cccc}
C_{2} & -S_{2} & 0 & 0 \\
0 & 0 & -1 & h_{2} \\
S_{2} & C_{2} & 0 & 0 \\
0 & 0 & 0 & 1
\end{array}\right] \\
& \underline{A_{3}^{2}}=\left[\begin{array}{cccc}
1 & 0 & 0 & 0 \\
0 & 0 & 1 & 0 \\
0 & -1 & 0 & 0 \\
0 & 0 & 0 & 1
\end{array}\right]\left[\begin{array}{cccc}
1 & 0 & 0 & 0 \\
0 & 1 & 0 & 0 \\
0 & 0 & 1 & d_{3} \\
0 & 0 & 0 & 1
\end{array}\right]=\left[\begin{array}{cccc}
1 & 0 & 0 & 0 \\
0 & 0 & 1 & d_{3} \\
0 & -1 & 0 & 0 \\
0 & 0 & 0 & 1
\end{array}\right] \\
& \underline{A_{4}^{3}}=\left[\begin{array}{cccc}
0 & 1 & 0 & 0 \\
-1 & 0 & 0 & 0 \\
0 & 0 & 1 & 0 \\
0 & 0 & 0 & 1
\end{array}\right]\left[\begin{array}{cccc}
C_{4} & -S_{4} & 0 & 0 \\
S_{4} & C_{4} & 0 & 0 \\
0 & 0 & 1 & 0 \\
0 & 0 & 0 & 1
\end{array}\right]=\left[\begin{array}{cccc}
S_{4} & C_{4} & 0 & 0 \\
-C_{4} & S_{4} & 0 & 0 \\
0 & 0 & 1 & 0 \\
0 & 0 & 0 & 1
\end{array}\right] \\
& \underline{A_{5}^{4}}=\left[\begin{array}{cccc}
0 & -1 & 0 & 0 \\
0 & 0 & 1 & 0 \\
-1 & 0 & 0 & 0 \\
0 & 0 & 0 & 1
\end{array}\right]\left[\begin{array}{cccc}
C_{5} & -S_{5} & 0 & 0 \\
S_{5} & C_{5} & 0 & 0 \\
0 & 0 & 1 & 0 \\
0 & 0 & 0 & 1
\end{array}\right]=\left[\begin{array}{cccc}
-S_{5} & -C_{5} & 0 & 0 \\
0 & 0 & 1 & 0 \\
-C_{5} & S_{5} & 0 & 0 \\
0 & 0 & 0 & 1
\end{array}\right] \\
& \underline{A_{6}^{5}}=\left[\begin{array}{cccc}
0 & 1 & 0 & 0 \\
0 & 0 & -1 & -h_{6} \\
-1 & 0 & 0 & 0 \\
0 & 0 & 0 & 1
\end{array}\right]\left[\begin{array}{cccc}
C_{6} & -S_{6} & 0 & 0 \\
S_{6} & C_{6} & 0 & 0 \\
0 & 0 & 1 & 0 \\
0 & 0 & 0 & 1
\end{array}\right]=\left[\begin{array}{cccc}
S_{6} & C_{6} & 0 & 0 \\
0 & 0 & -1 & -h_{6} \\
-C_{6} & -S_{6} & 0 & 0 \\
0 & 0 & 0 & 1
\end{array}\right]
\end{aligned}
$$


and the HTM of end effector ${ }^{0} T_{6}$ is calculated as:

$$
\underline{T_{6}^{0}}=\underline{A_{1}^{0} A_{2}^{1} A_{3}^{2} A_{4}^{3} A_{5}^{4} A_{6}^{5}}=\left[\begin{array}{cccc}
n_{x} & s_{x} & a_{x} & p_{x} \\
n_{y} & s_{y} & a_{y} & p_{y} \\
n_{z} & s_{z} & a_{z} & p_{z} \\
0 & 0 & 0 & 1
\end{array}\right]
$$

Where,

$$
\begin{gathered}
n_{x}=C_{6}\left[S_{4} S_{1}-C_{4} C_{2} C_{1}\right]-S_{6}\left[S_{5} C_{4} S_{1}+C_{1} C_{2} S_{4}\right]-C_{1} C_{5} S_{\partial 2}, \\
n_{y}=S_{6}\left[S_{5} C_{1} C_{4}-S_{1} C_{2} S_{4}+C_{5} S_{1} S_{2}\right]-C_{6}\left[S_{4} C_{1}-C_{2} S_{1} C_{4}\right], \\
n_{z}=-S_{6}\left[C_{2} C_{5}+S_{2} S_{4} S_{5}\right]-S_{2} C_{4} C_{6} \\
s_{x}=-C_{6}\left[S_{5}\left(C_{4} S_{1}+S_{4} C_{1} C_{2}\right)-C_{1} C_{5} S_{2}\right]-S_{6}\left[S_{4} S_{1}-C_{1} C_{2} C_{4}\right], \\
s_{y}=C_{6}\left[S_{5}\left(C_{4} C_{1}-S_{4} C_{2} S_{1}\right)+S_{2} C_{5} S_{1}\right]+S_{6}\left[S_{4} C_{1}+C_{4} C_{2} S_{1}\right], \\
s_{z}=-C_{6}\left[C_{2} C_{5}+S_{2} S_{5} S_{4}\right]+S_{2} C_{4} S_{6} \\
a_{x}=C_{5}\left(S_{1} C_{4}+C_{1} S_{4} C_{2}\right)+C_{1} S_{2} S_{5} \\
a_{y}=S_{1} S_{2} S_{5}-C_{5}\left(C_{1} C_{4}-C_{2} S_{1} S_{4}\right) \\
a_{z}=S_{2} S_{4} C_{5}-C_{2} S_{5} \\
p_{x}=h_{2} S_{1}+h_{6}\left[C_{5}\left(C_{4} S_{1}+C_{1} C_{2} S_{4}\right)+C_{1} S_{2} S_{5}\right]-d_{3} C_{1} S_{2} \\
p_{y}=-h_{2} C_{1}-h_{6}\left[C_{5}\left(C_{1} C_{4}-C_{2} S_{1} S_{4}\right)-S_{1} S_{2} S_{5}\right]-d_{3} S_{1} S_{2} \\
p_{z}=h_{1}-h_{6}\left(C_{2} S_{5}-C_{5} S_{2} S_{4}\right)+d_{3} C_{2}
\end{gathered}
$$

\subsection{Comparing the Results}

We employed both D-H and Convenient approach to compare the two methods in kinematics analysis of Stanford manipulator and indicate the advantages of our proposed method clearly. Comparing the homogenous transformation matrix of the end effector, ${ }^{0} T_{6}$ in equations (14), (17) and, we can see that all elements forming the matrix are exactly equal in all equations.

It proves a significant meaning that regardless of what coordinate system is used, the end-effector position and orientation would be the same using both methods.

Using a different coordinate system in convenient approach, although the individual HTMs for joints 1 to 6 are different, as long as the first and last coordinate systems are similar, their multiplication forming the end effector HTM is the same as previous. It means that in convenient approach we have the freedom to choose our own arbitrary coordinate system with only one simple rule to be implemented: the $\mathrm{Zi}$ axis must lie along the movement of joint $i$ degree of freedom. Therefore, the devastating steps of coordinate system assignment and link/joint parameters extraction in D-H method will no longer is needed. This is a prefect reason for priority of "the Convenient Approach" over the conventional D-H representation

\section{CONCLUTIONS}

In this paper, Relative Transformation Principle (RTP) has been thoroughly introduced with clear concept and example, then a new simplified and accessible method called "The Convenient Approach" for the solution of robot kinematics has been developed. The advantages of this approach has been discussed by comparing with the well-known D-H representation through performing the forward kinematic on Stanford industrial robot. The results showed the consistency of the new method with much more simple assignment of the coordinate framed. The authors believe that by strong basis of principle and easy approach of assignment, this method would become a very helpful tool in robotics education and engineering. 


\section{REFERENCES}

[1] Reuleaux F, Ferguson ES. Kinematics of machinery: outlines of a theory of machines: Courier Corporation; 2012.

[2] Denavit J. A kinematic notation for lower-pair mechanisms based on matrices. Trans of the ASME Journal of Applied Mechanics. 1955;22:215-21.

[3] Paul RP, Shimano B, Mayer GE. Kinematic control equations for simple manipulators. IEEE Transactions on Systems, Man, and Cybernetics. 1981;11:449-5.

[4] Paul RP, Shimano B, editors. Kinematic control equations for simple manipulators. Decision and Control including the 17th Symposium on Adaptive Processes, 1978 IEEE Conference on; 1979: IEEE.

[5] Lee CG. Robot arm kinematics, dynamics, and control. Computer. 1982;15(12):62-80.

[6] Craig JJ. Introduction to robotics: mechanics and control: Pearson Prentice Hall Upper Saddle River; 2005.

[7] Gonzalez RC, Fu K, Lee C. Robotics: Control, Sensing, Vision, and Intelligence. Editora Hardcover. 1987.

[8] Paul R. Robot manipulators: mathematics, programming and control, 1981. MIT Press.28:307-16.

[9] Lee CG, Ziegler M. Geometric approach in solving inverse kinematics of PUMA robots. Aerospace and Electronic Systems, IEEE Transactions on. 1984(6):695-706.

[10] Koren Y, Koren Y. Robotics for engineers: McGraw-Hill New York et al; 1985.

[11] Siciliano B, Sciavicco L, Villani L, Oriolo G. Robotics: modelling, planning and control: Springer Science \& Business Media; 2010.

[12] Tsai L-W. Robot analysis: the mechanics of serial and parallel manipulators: John Wiley \& Sons; 1999.

[13] Crane III CD, Duffy J. Kinematic analysis of robot manipulators: Cambridge University Press; 2008. 